\title{
Review: angiotensin converting enzyme inhibitors are beneficial in type 1 diabetes mellitus and microalbuminuria regardless of baseline risk factor status
}

\author{
The ACE Inhibitors in Diabetic Nephropathy Trialist Group. Should all patients with type 1 diabetes mellitus and \\ microalbuminuria receive angiotensin-converting enzyme inhibitors? A meta-analysis of individual patient data. Ann \\ Intern Med 2001 Mar 6;134:370-9. \\ QUESTION: In patients with type 1 diabetes mellitus and microalbuminuria, what is the \\ effect of angiotensin converting enzyme (ACE) inhibitors independent of confounding \\ variables?
}

\section{Data sources}

Studies were identified by searching Medline and bibliographies of relevant papers and by contacting authors.

\section{Study selection}

Studies were selected if they included $\geqslant 10$ patients with type 1 diabetes and microalbuminuria (defined as having an albumin excretion rate of 20 to 200 $\mu \mathrm{g} /$ minute, or approximately 30 to $300 \mathrm{mg} /$ day, who were judged not to be hypertensive at the time of the original trial), had a placebo or non-intervention control group, had follow up $\geqslant 1$ year, and measured albumin excretion rate at baseline and at $\geqslant 1$ follow up visit.

\section{Data extraction}

Authors were contacted to provide individual patient data on age, duration of diabetes, sex, baseline treatment assignment, albumin excretion rate at baseline and follow up, blood pressure, and glycaemic control.

\section{Main results}

12 studies (698 patients, mean age range 32 to 48 y) met the selection criteria. The modal follow up duration was 2 years (range 1-4 y). 4 studies used lisinopril, 3 used captopril, 2 used enalapril, 2 used ramipril, and 1 used perindopril.

Analyses are presented for 2 year outcomes by using 10 studies (646 patients) that had $\geqslant 2$ years of follow up. ACE inhibitors were more effective than placebo for reducing progression to macroalbuminuria (odds ratio [OR] $0.38,95 \%$ CI 0.25 to $0.57, \mathrm{p}<0.001)$ and increasing regression to normoalbuminuria (OR 3.07, CI 2.15 to 4.44, $\mathrm{p}<0.001)$. After adjustment for baseline albumin excretion rate, the mean albumin excretion rate was $50.5 \%$ (CI $29.2 \%$ to $65.5 \%, \mathrm{p}<0.001$ ) lower in patients receiving ACE inhibitors than in those receiving placebo. Patients with baseline albumin excretion rates at the upper boundary of microalbumin excretion $(200 \mu \mathrm{g} / \mathrm{min})$ had a higher 2 year difference between treatment and placebo groups in albumin excretion rate than those with baseline albumin excretion rates at the lower boundary of $20 \mu \mathrm{g} / \mathrm{min}$ (relative rate reduction $74.1 \% v 17.8 \%, \mathrm{p}=0.04$ ). Adjustment for change in blood pressure attenuated the treatment effect to a $45.1 \%$ relative rate reduction (CI $18.6 \%$ to $63.1 \%$, $\mathrm{p}<0.001)$. No differences in treatment effects were seen according to baseline risk factors of age, sex, duration of diabetes, haemoglobin $A_{1 c}$ concentration, or diastolic blood pressure.

\section{Conclusions}

In patients with type 1 diabetes mellitus and microalbuminuria, angiotensin converting enzyme (ACE) inhibitors reduce progression to macroalbuminuria and increase regression to normoalbuminuria. Beneficial treatment effects are greatest for those with the highest levels of baseline microalbuminuria, but no difference exists according to other baseline risk factors. Changes in systemic blood pressure from ACE inhibitors explains some, but not most, of the benefits.

\section{COMMENTARY}

Partly because of results of individual randomised trials included in this meta-analysis, evidenced based clinical practice guidelines recommend the use of ACE inhibitors for patients with type 1 diabetes and microalbuminuria. Although this review does not change the guideline recommendations, it does answer important questions about these patients that were not answered in the individual trials. In particular, should patients with minimal microalbuminuria be treated, and can microalbuminuria be reversed?

This meta-analysis aggregated individual patient data from each trial rather than from the published results. This method allows for a more reliable analysis of the overall effect of ACE inhibitors on microalbuminuria and assessment of its effects based on specific patient characteristics in a sample larger than that attained in any single trial. The value of arresting, if not reversing, increased albumin excretion rates depends on the argument, accepted by many, ${ }^{1}$ that it is a valid surrogate for progression to clinically important diabetic nephropathy. However, an estimate of the number of patients with microalbuminuria needed to treat to prevent, for example, 1 person from developing macroalbuminuria would be beneficial for weighing the clinical importance against the cons of long term ACE inhibitor use.

I confess I have previously only observed otherwise well young people with type 1 diabetes who had low but abnormal albumin excretion rates (eg, 30 to $50 \mathrm{mg} / \mathrm{d}$ ) and blood pressure concentrations $<130 / 80 \mathrm{~mm} \mathrm{Hg}$ who opted to begin ACE inhibition when these measurements rose. Given the results of this meta-analysis, I will now be much less inclined to do this.

Jeffrey L Mahon, MD, MSc University of Western Ontario

London, Ontario, Canada

1 Meltzer S, Leiter L, Daneman D, et al. 1998 clinical practice guidelines for the management of diabetes in Canada. Canadian Diabetes Association. CMAJ 1998;159:S1-29. 